rq

\author{
دراسة اقتصاديه لاستجابة العرض لمحصول القمح في جمهورية مصر العربية

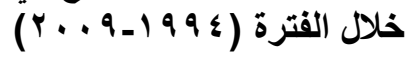 \\ شهيناز عيد محمود \\ قسم الاقتصاد الزراعى - كلية الزراعة - جامعة الفيوم - مصر
}

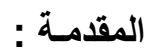

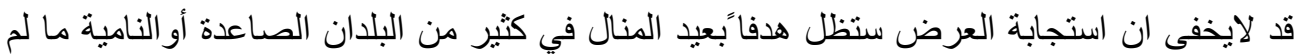

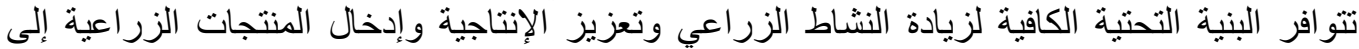

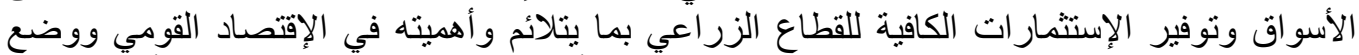

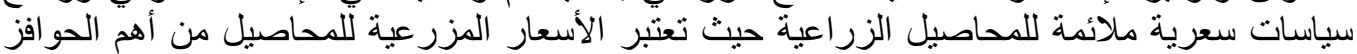

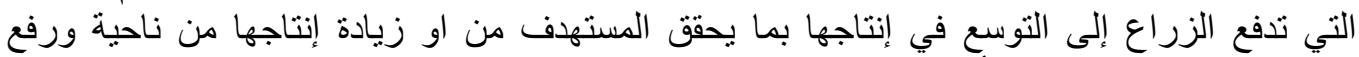
المسنوى المعيشي من ناحية أخرى النئ.

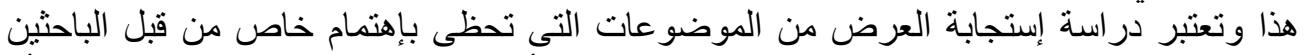

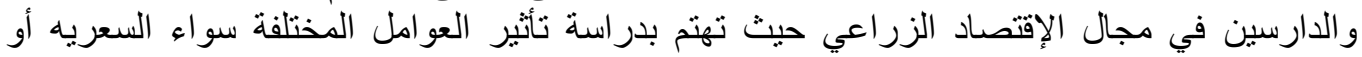

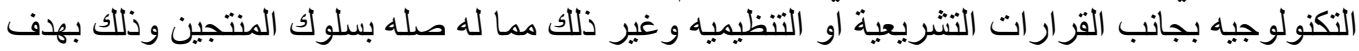

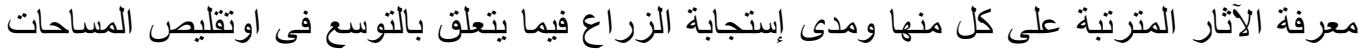

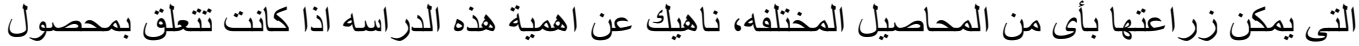

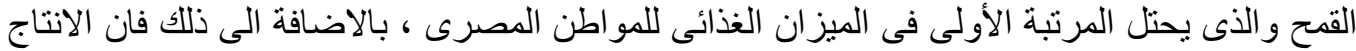

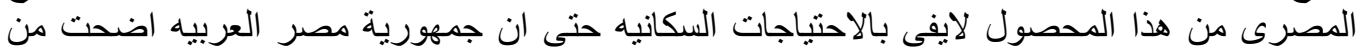

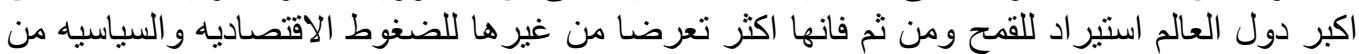

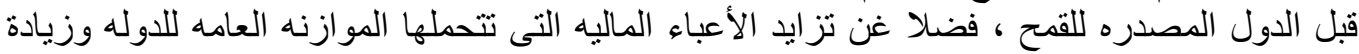

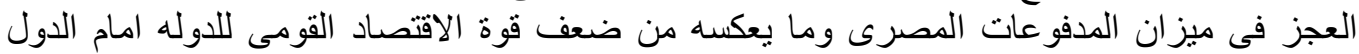

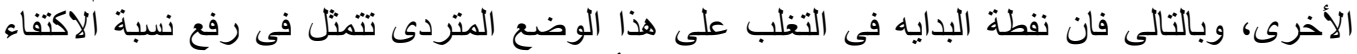

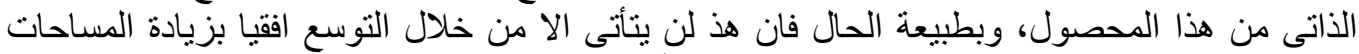

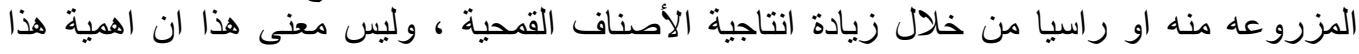

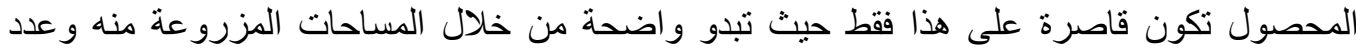
العاملين فى مجال انتاجه او تصنيعه اوتسويقه او تمويله الى غير ذلى ذلك من الانشطة المنعلقة بهذا

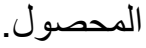

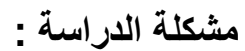

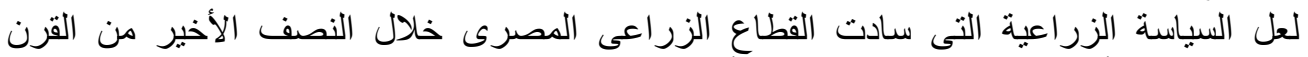

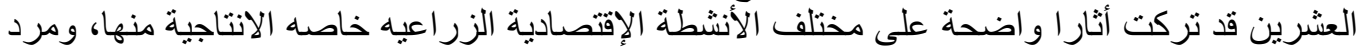

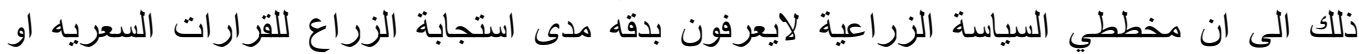

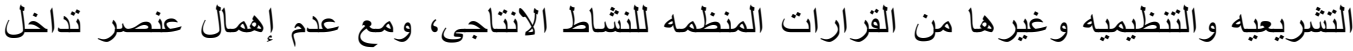

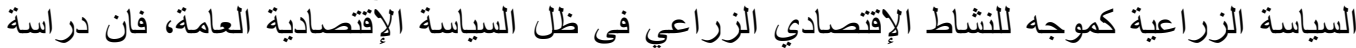

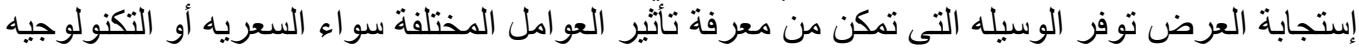

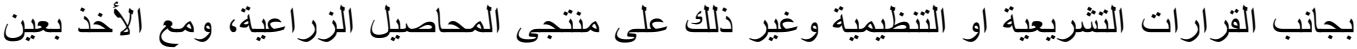

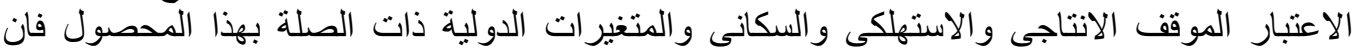

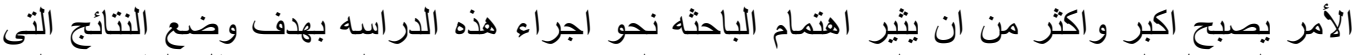

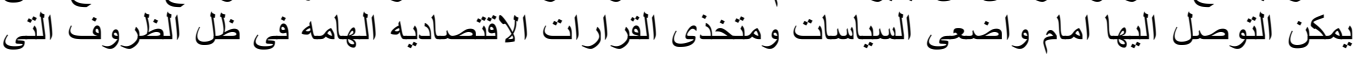

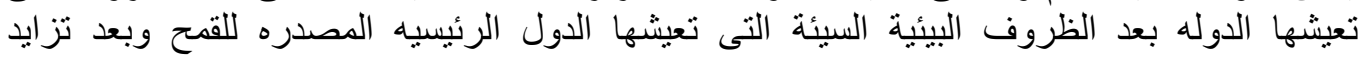

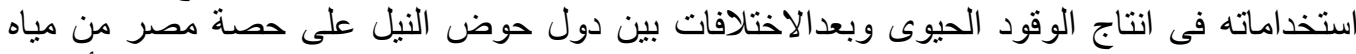

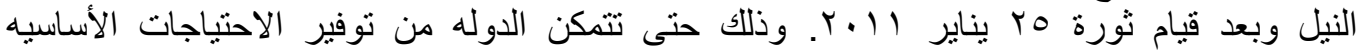
لمو اطنيها دون تعرض لضغورة نور خارجيه ايا كانت طبيعتها.

Fayoum J. Agric. Res. \& Dev., Vol.25, No.2, July, 2011 


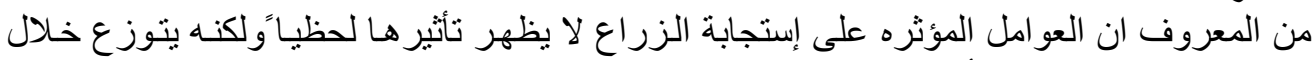

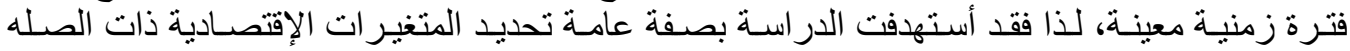

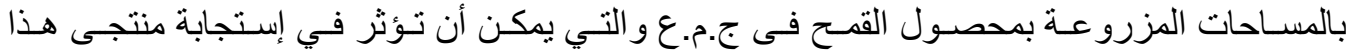

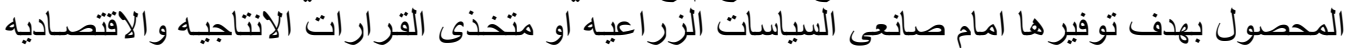

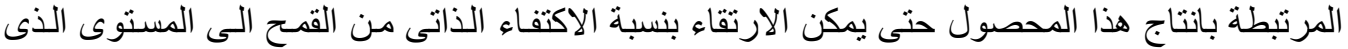

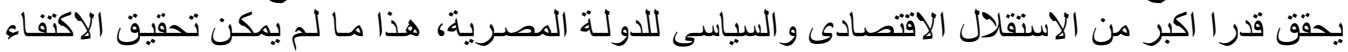

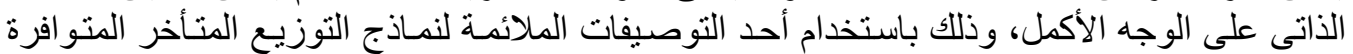

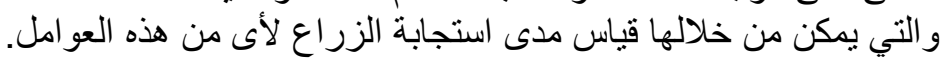

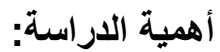

تكتسب هذه الدر اسة أهميتها من خلال ما سبقت الاشارة اليه في المقدمة عن اهمية هذا الاقتصسادى مئه

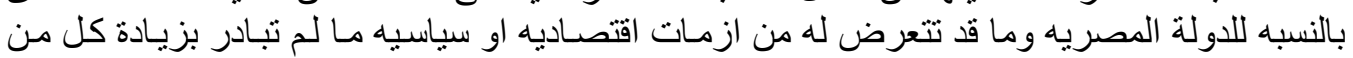

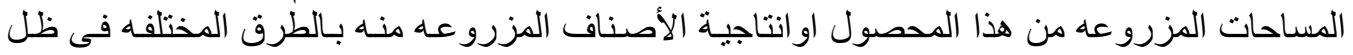
الظروف الانتاجيه و الاستهلاكيه المصنه هذرية. الطريقة البحثية ومصادر البيانات:

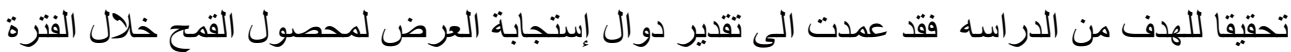

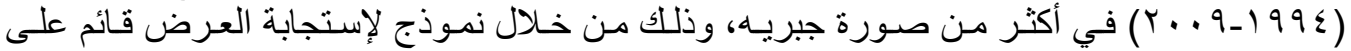

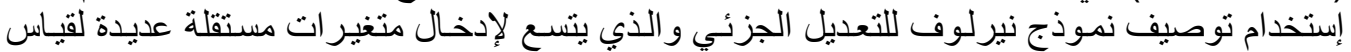

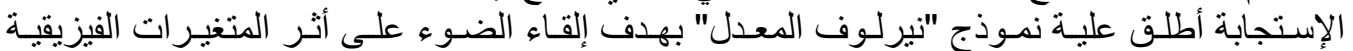

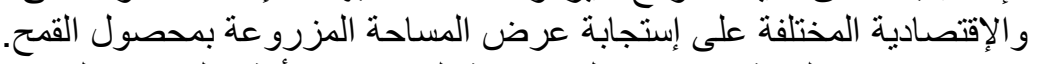

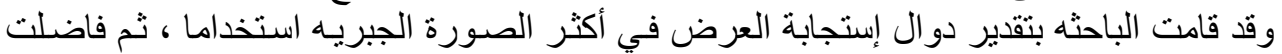

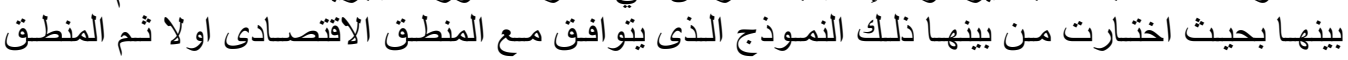

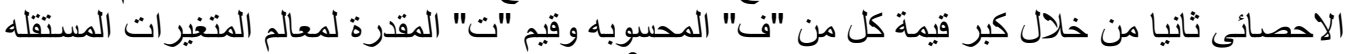

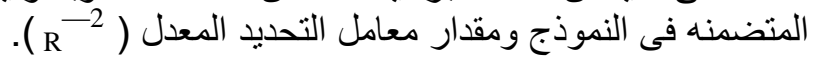

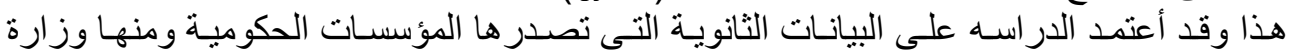

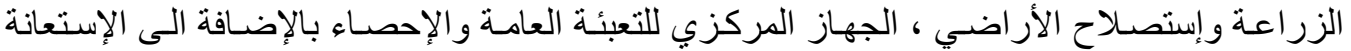

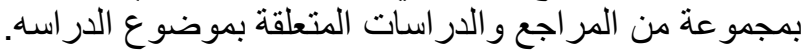

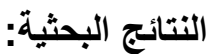

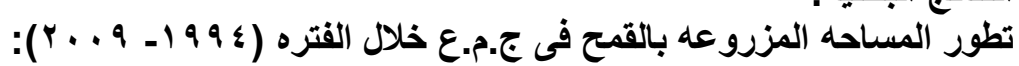

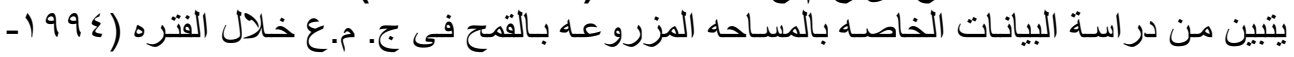

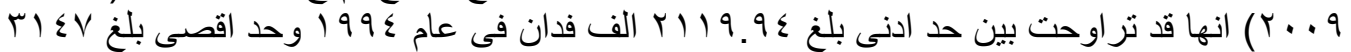

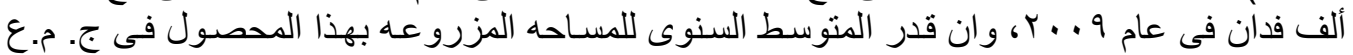

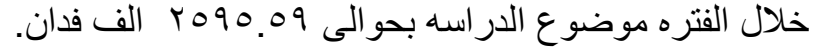

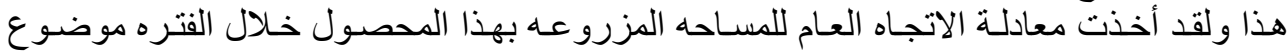
الدر اسه الصوره التاليه : س **(0.7 .0$) * *(Y \leq . \wedge, Y)$ ف $. T V M=r-, * * r), \Lambda T V=$,

حيث تشير صى الى المساحه التقديريه المزرو عه بالقمح فى السنه س، س تشير الى الزمن، هـ =

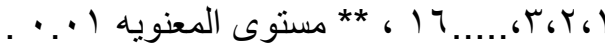

Fayoum J. Agric. Res. \& Dev., Vol.25, No.2, July, 2011 
I

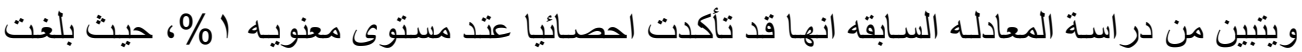

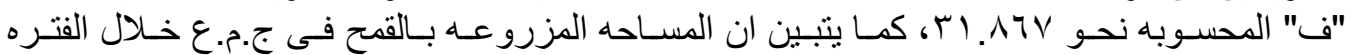

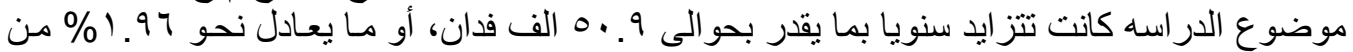

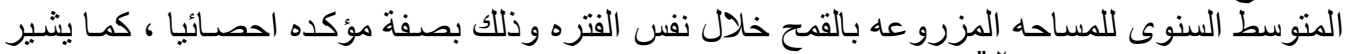

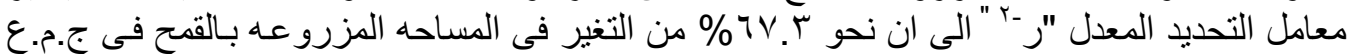

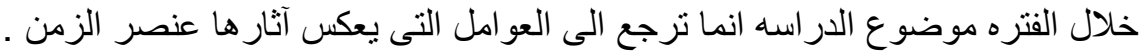

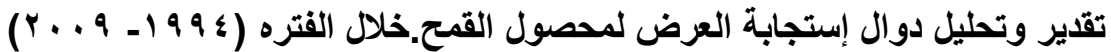

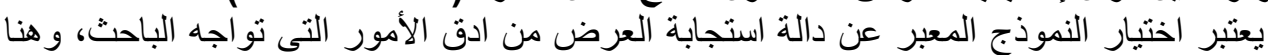

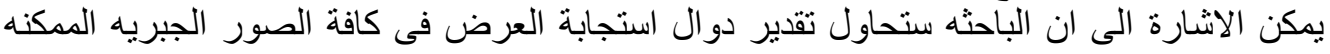

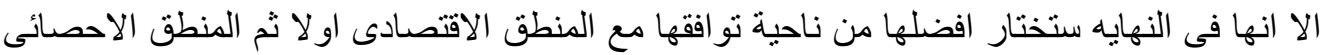

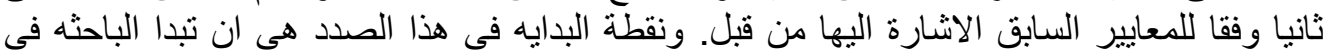

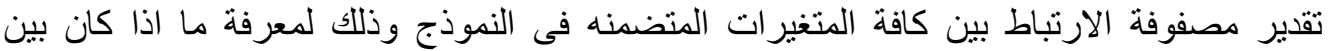

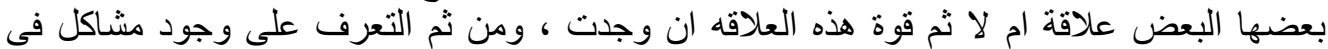

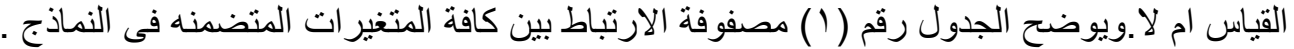

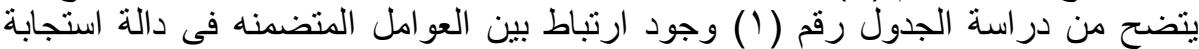

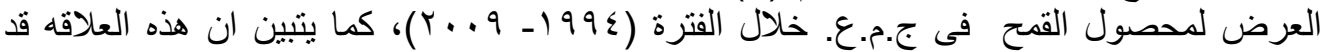

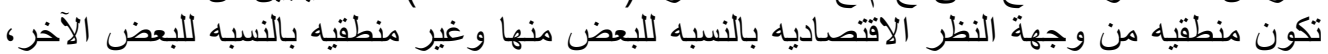

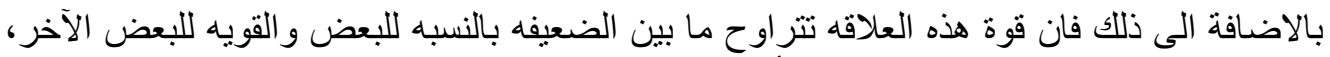

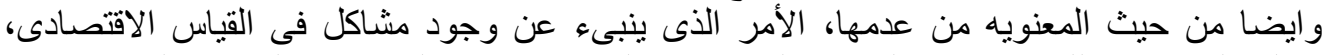

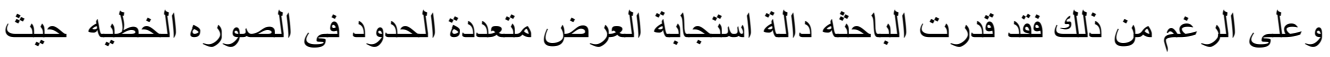
كانت على الصوره التالية:

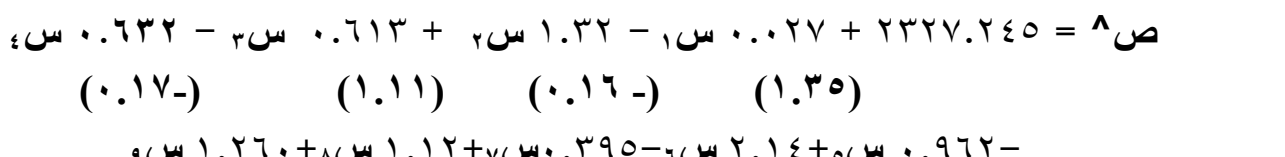

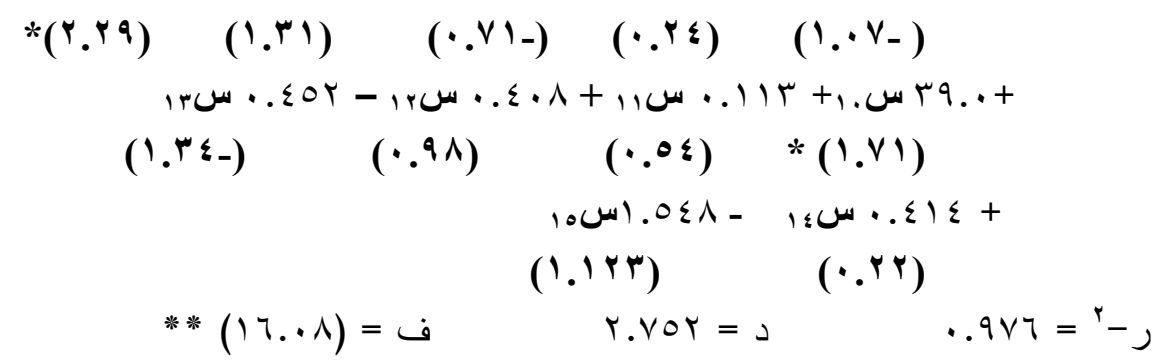

Fayoum J. Agric. Res. \& Dev., Vol.25, No.2, July, 2011 


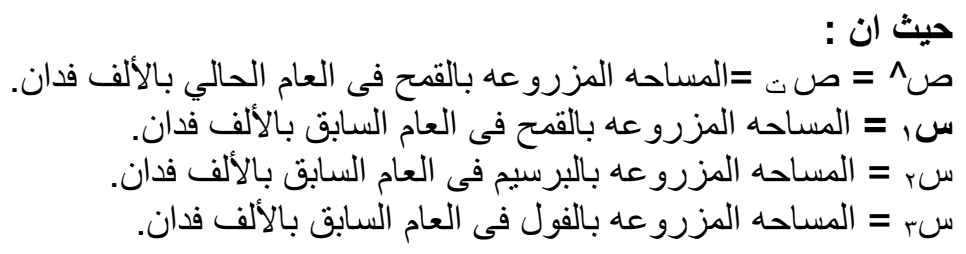

Fayoum J. Agric. Res. \& Dev., Vol.25, No.2, July, 2011 
س؛ = المساحه المزروعه بالشعير فى العام السابق بالألف فدان.

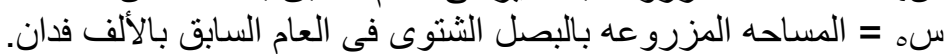
س = = انتاجية الفدان من القمح بالأردب في العَ العام السابق.

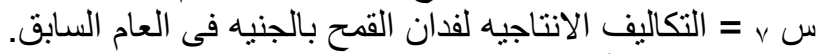

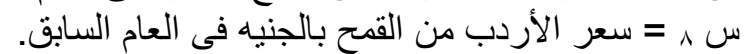
س ه = السعر العالمى لأردب القمح بالجنيه في العنى العام السابق.

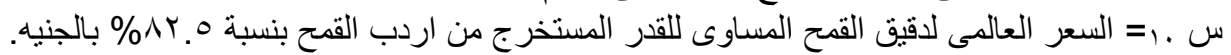

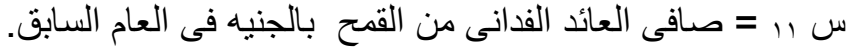

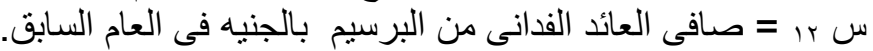

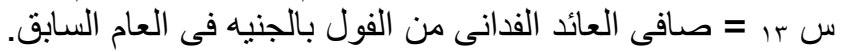

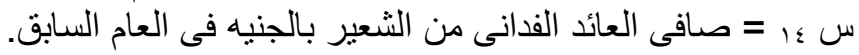

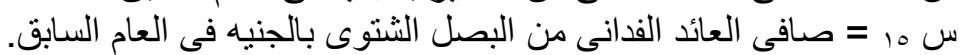

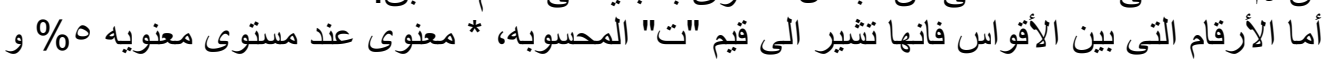

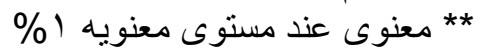

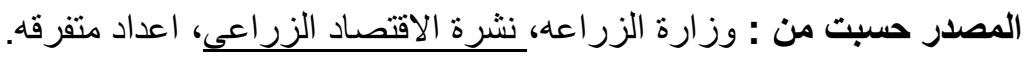

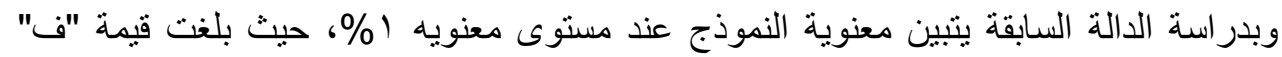

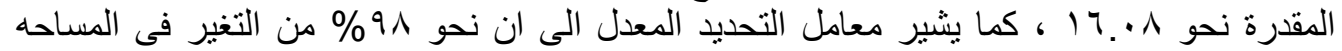

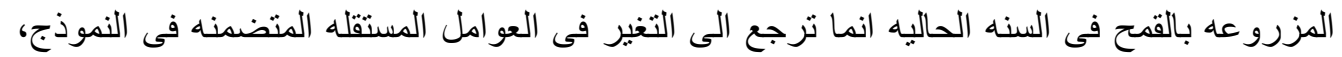

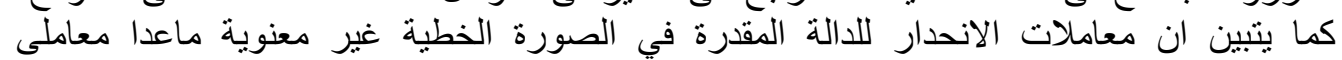

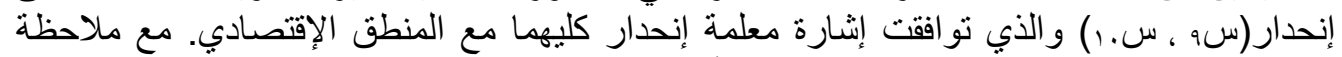

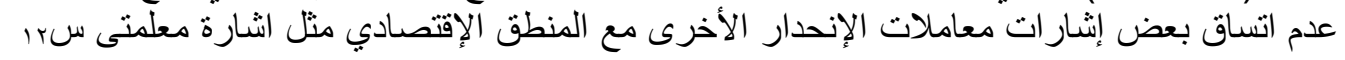
هذا وعلى الرغم من الملاحظات السابق فان الباحثه قد عمدت الى تفدبر دالة استجابة العرض فئل

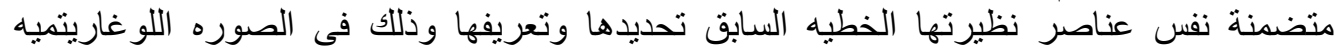
المزدوجه، فكانت على الصوره التاليه:

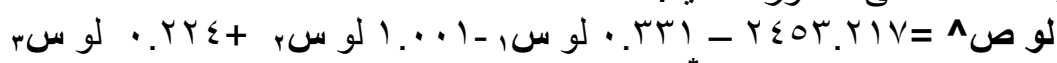
$(1 . \varepsilon Y) \quad(. \vee \wedge-) \quad\left(Y . \varepsilon q^{*}-\right)$

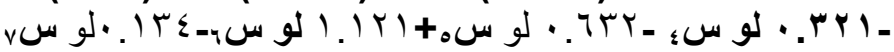

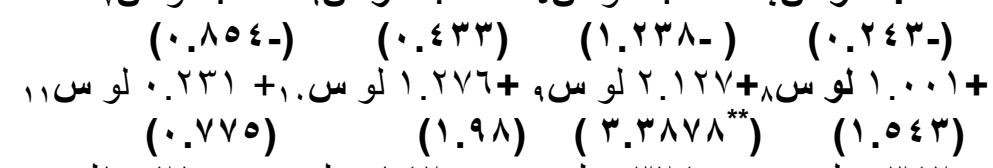
+

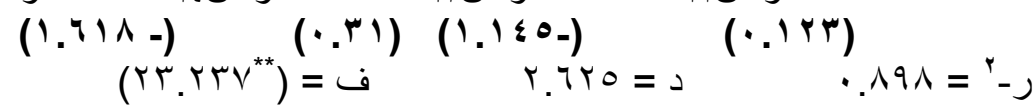

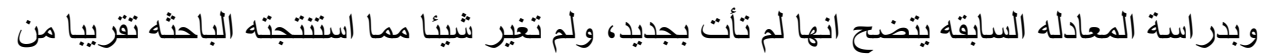

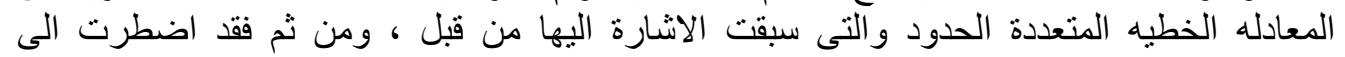

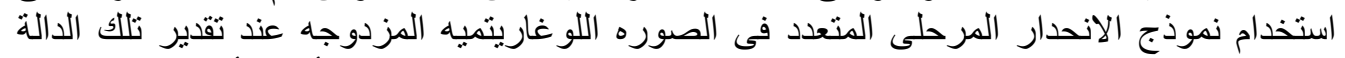

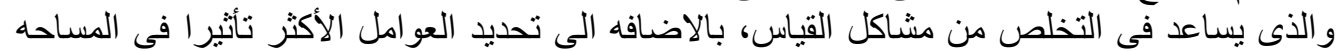

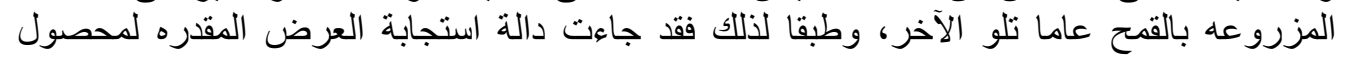

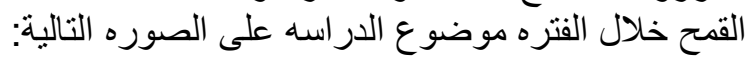

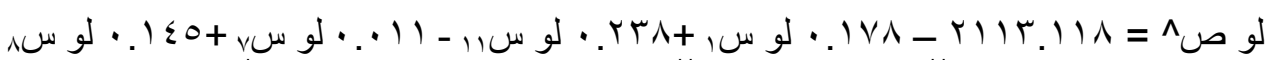

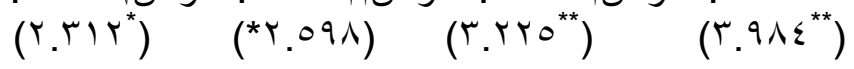

Fayoum J. Agric. Res. \& Dev., Vol.25, No.2, July, 2011 


$$
\varepsilon r, 70 \Lambda^{* *}=0 \quad \text { l. ror }=د \quad \cdot 917={ }^{r-} \text { ر }
$$

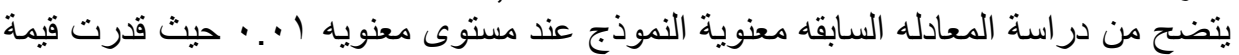

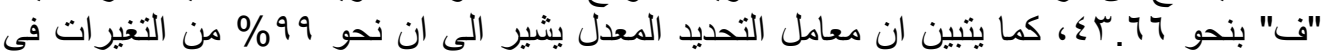

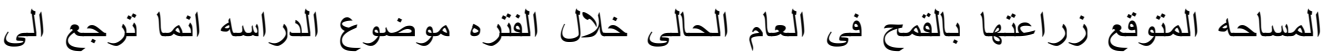

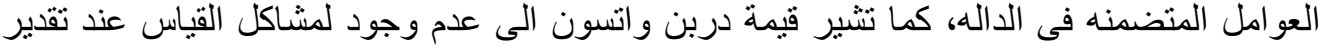

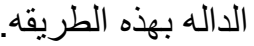

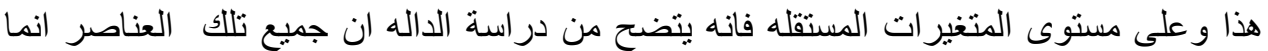

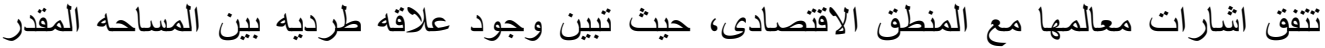

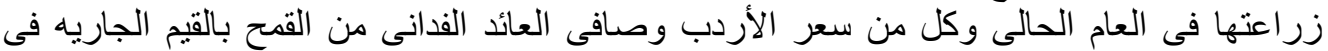

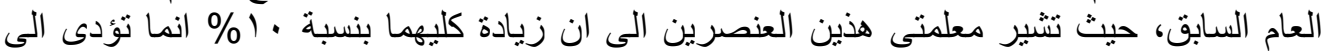

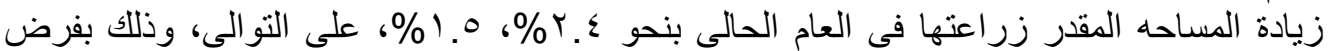

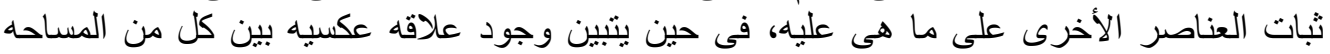

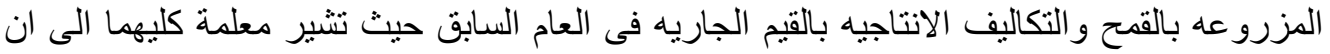

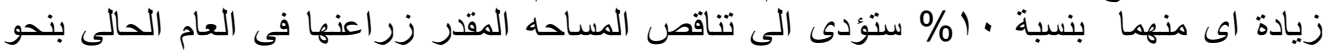

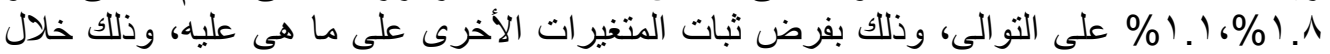

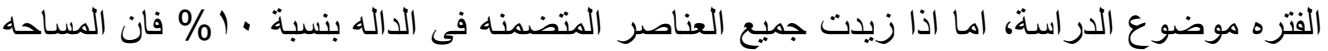

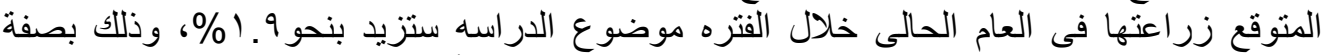

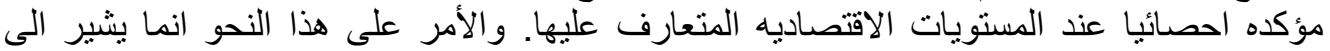

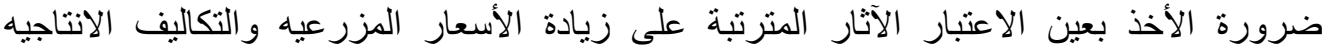

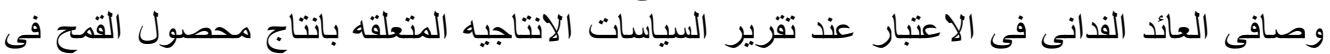

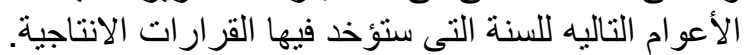

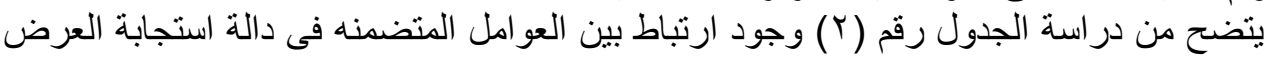

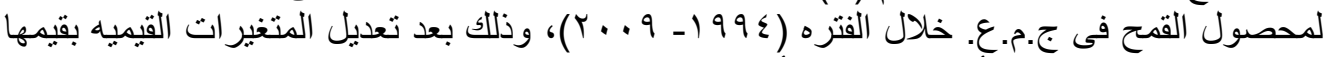

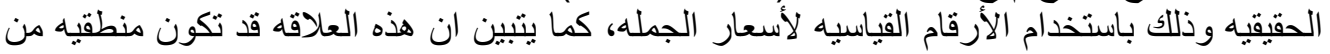

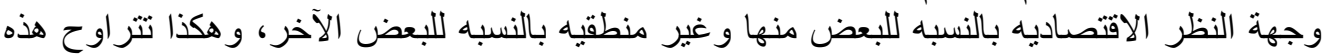

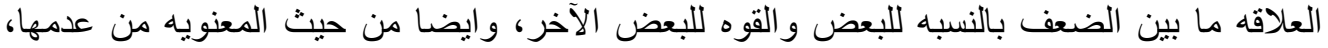

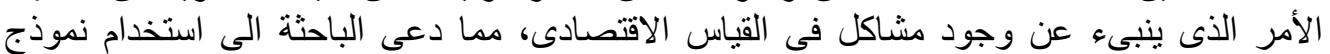

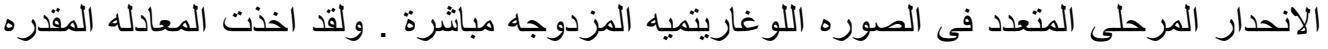

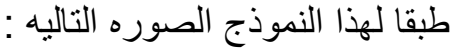

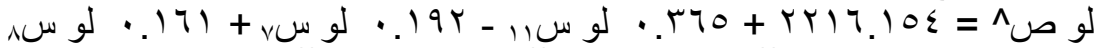

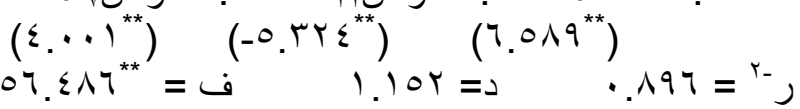

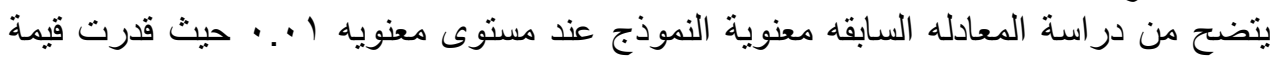

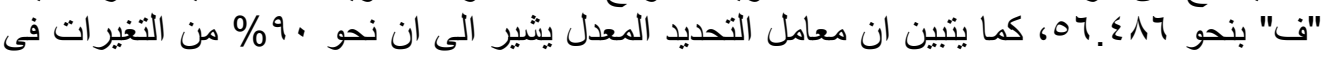

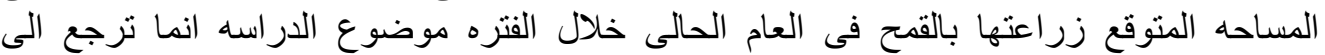

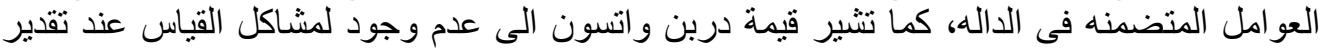


هذا و على مستوى المتغير ات المستقله فانه يتضح من دراسة الداله ان جميع تللك العناصر انما

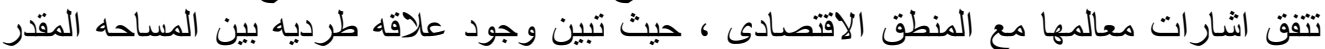

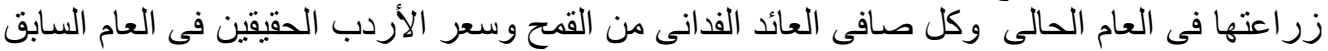

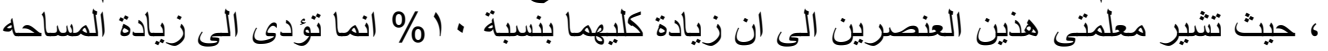

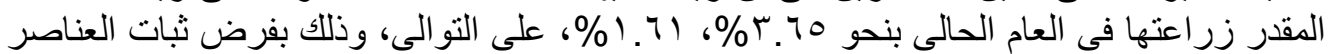

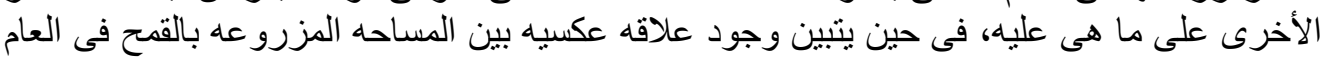

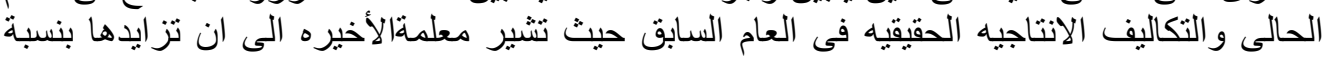

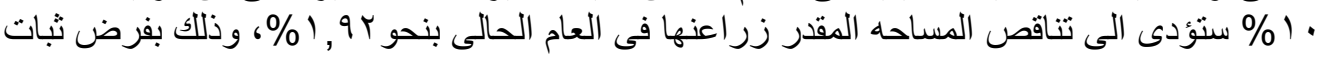

Fayoum J. Agric. Res. \& Dev., Vol.25, No.2, July, 2011 


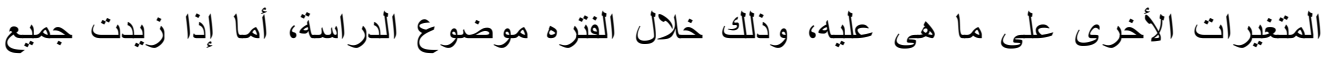

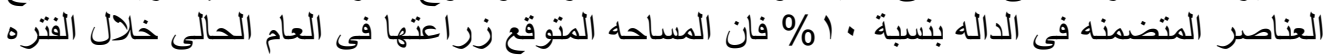

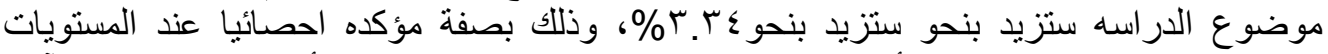

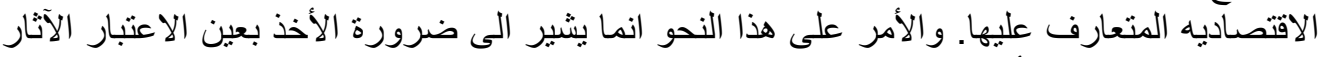

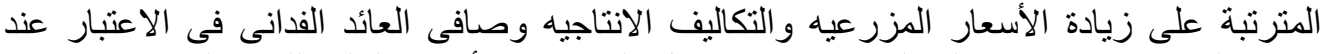

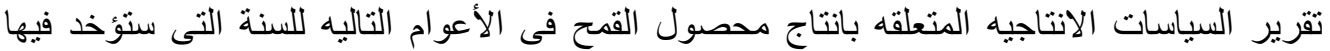

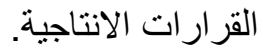

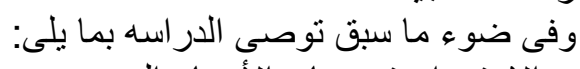

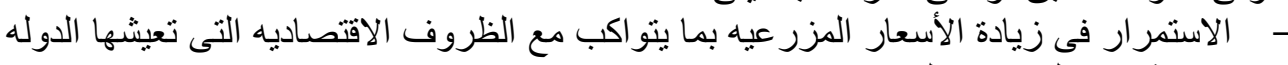

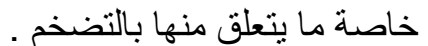

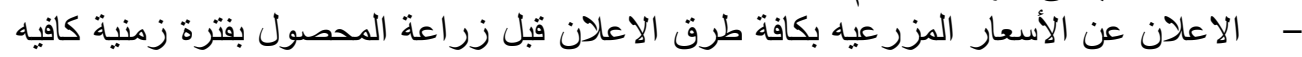

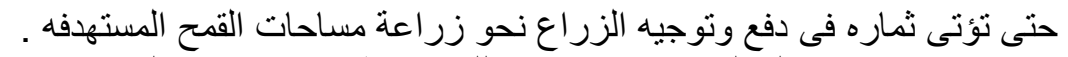

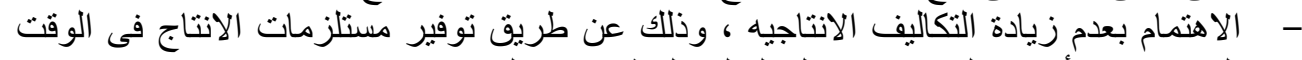

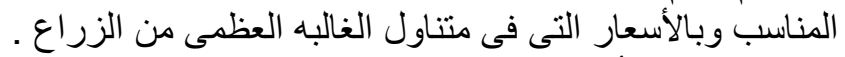

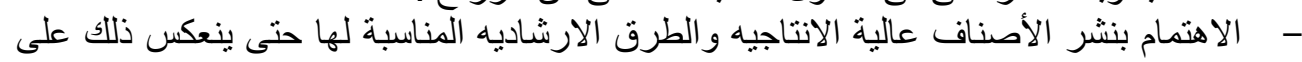
صافى العائد الفدانى بالزياده.

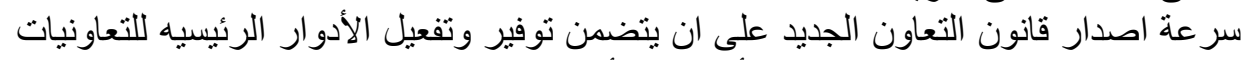
الاتتاجيه و التسويقيه منها التى تحقق الأهداف المأمولة منها.

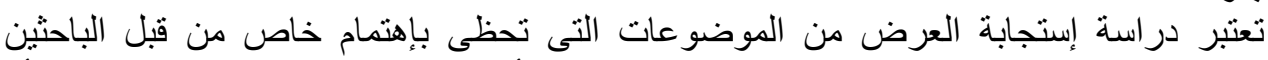

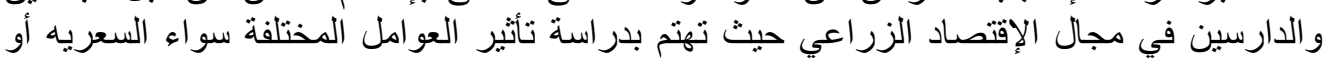

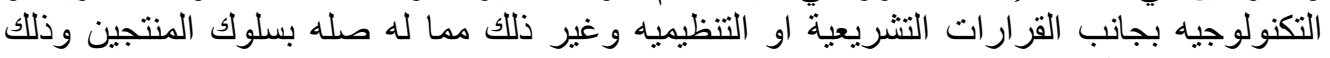

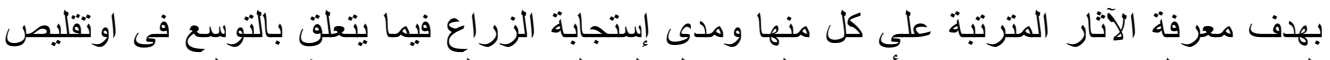

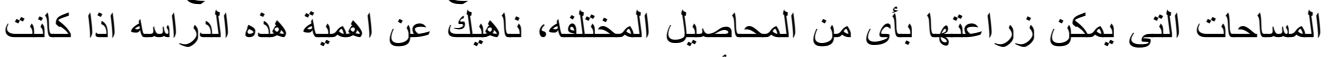

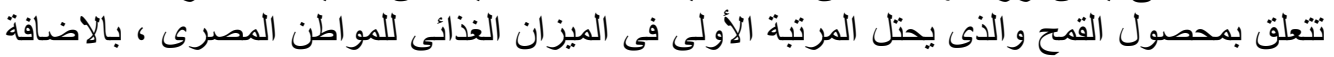

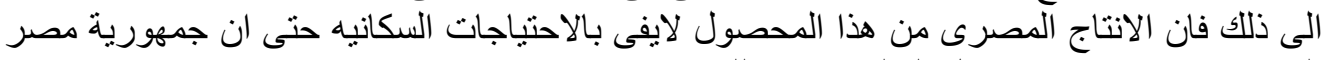

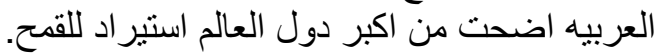

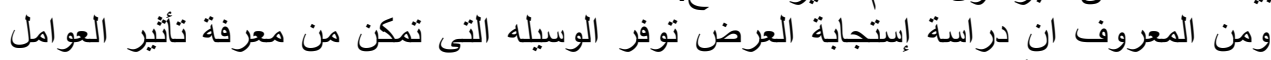

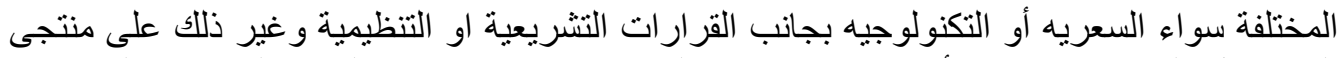

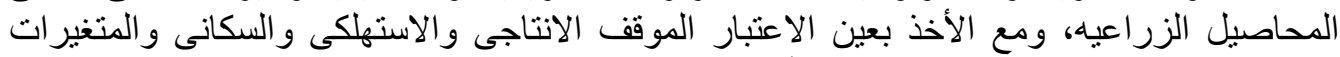

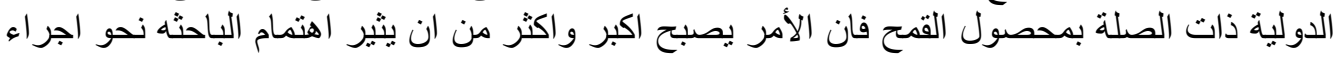

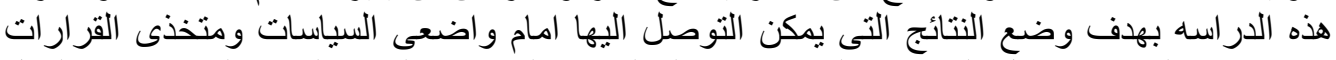

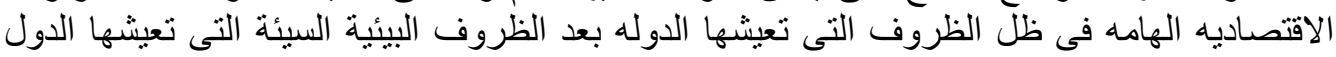

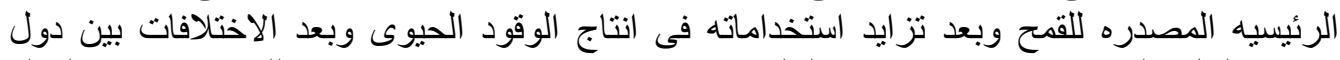

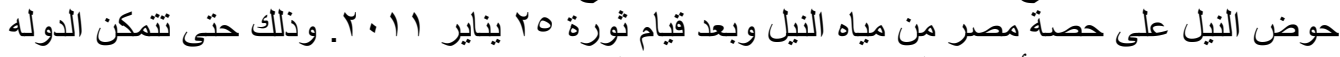

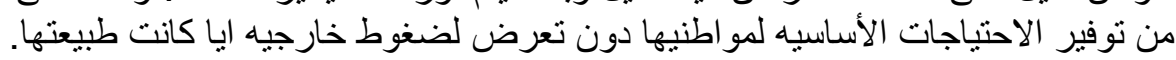

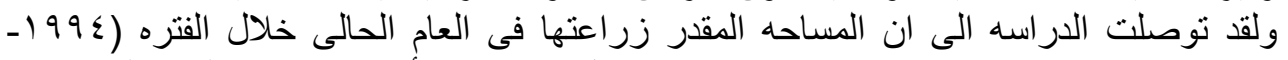

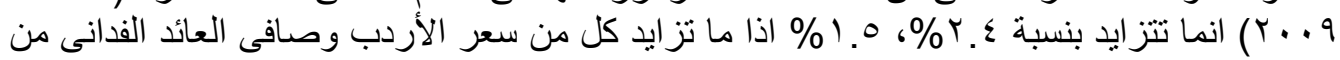

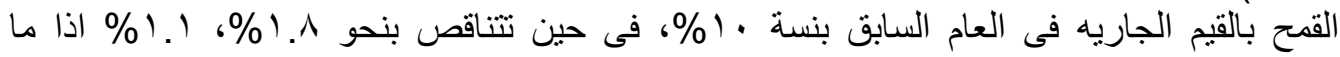

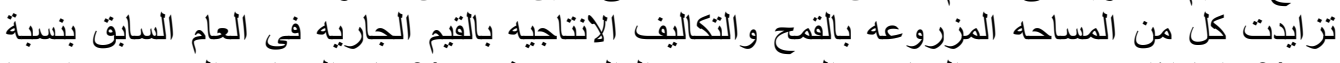

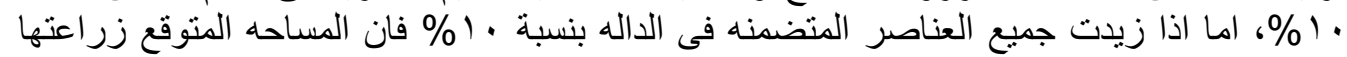

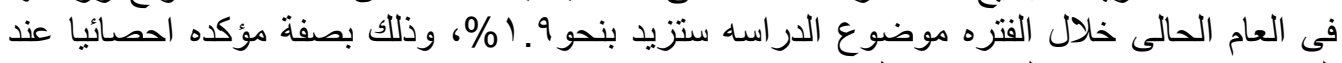
المستويات الاقتصاديه المتعارف علئ عليها.

Fayoum J. Agric. Res. \& Dev., Vol.25, No.2, July, 2011 
rv

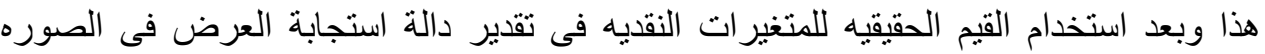

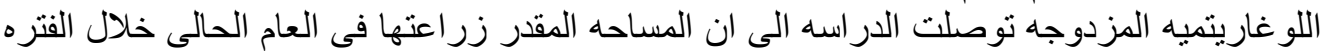

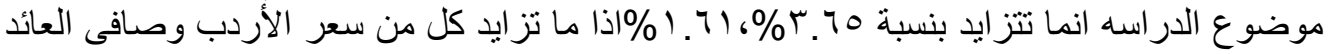

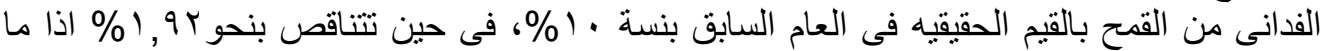

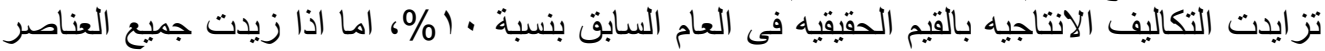

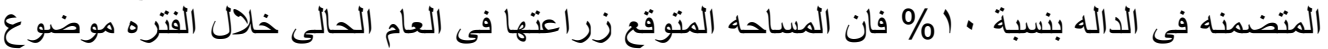

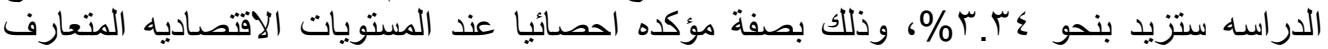

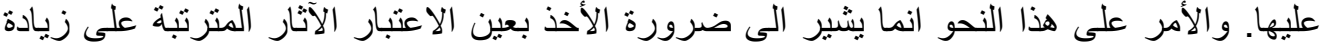

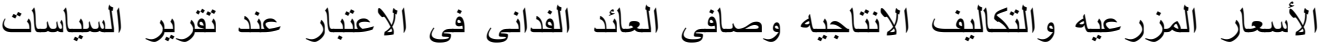
الانتاجيه المتعلقه بانتاج محصول القمح فى الأعو ام التاليه للسنة التى ستؤخد فيها القرار ات التهات الانتاجيه.

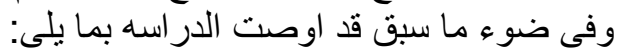

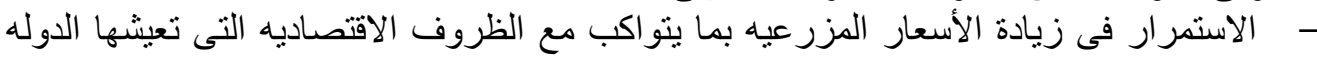

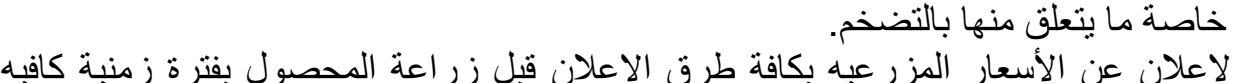

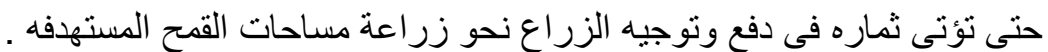

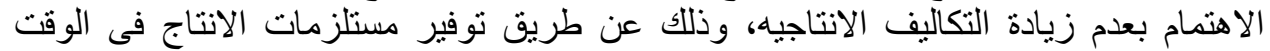

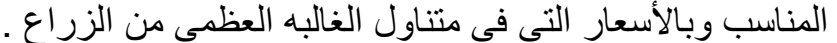

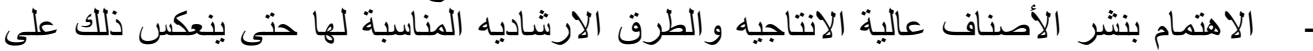
صافى العائد الفدانى بالزياده. ـ سرعة اصدار قانون التعاون الجديد على ان يتضمن توفير وتفعيل الأدوار الرئيسيه للتعاونيات

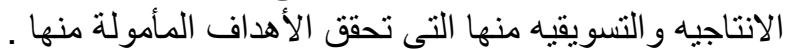

ا - - الجهاز: الجهاز المركزي للتعبئة العامة والإحصاء، "الكتاب الاحصائي السنوي"، أعداد متفرقة.

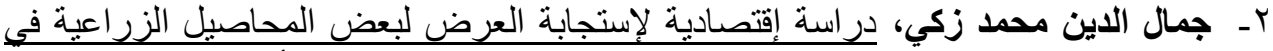

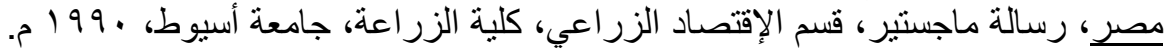

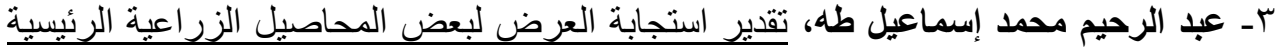

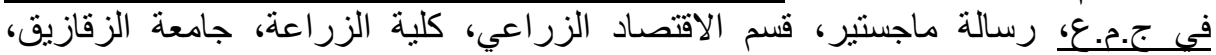
. 910 ـ - محمد كامل ابراهيم ريحان، دكتور، "نماذج استجابة العرض لأهم حاصلات الخضر و الفاكهة

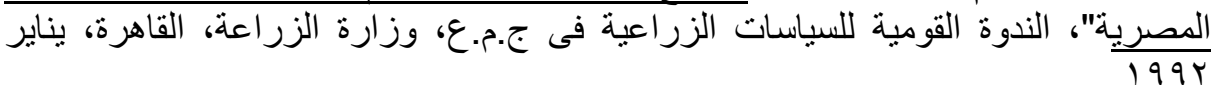
ــ نعمان مسعد أبو سمرة، تقدير استجابة العرض لبعض الحاصلات الزر اعية في محافظة

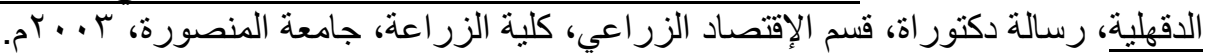

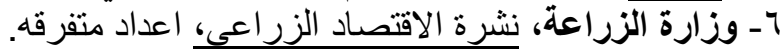

7- Nerlov,Mark,Leon " Estimates of Elasticities of Supply of Selected Agricultural Commodities" J.Farm Econ., Vol.38,No.2,May,1956,PP,496509.

\section{AN ECONOMIC STUDY FOR SUPPLY RESPONSE OF WHEAT CROP IN A.R.E .AT (1994 - 2009)}

\section{ABSTRACT}

Fayoum J. Agric. Res. \& Dev., Vol.25, No.2, July, 2011 
The study of supply response offers the methodology that enables identifying the effects of different factors either price or technological, in conjunction with legislative or regulatory ruling and others on wheat producers. Taking into consideration the production, consumption, demographic position, and the global changes that are related to this product, makes this subject more interesting for researchers to perform this study aiming to provide analysis that may be offered for political and economical decision makers.

Studies concluded that the estimated area for agriculture between (1994 2009) is increasing by $2.4 \%$ and $1.5 \%$ if the increase in Erdab price and net Feddan of wheat returns of current values in the previous year is $10 \%$, whereas the area for agriculture decreases by $1.8 \%$ and $1.1 \%$ if the increase in area planted of wheat and production costs of current values in the previous year is $10 \%$. However, if all the parameters in the function increased by $10 \%$, the estimated planted area in the year under study would increase by about $1.9 \%$ which is statistically confirmed at all known economical standards.

This is achieved in light of using the real values for the monetary changes in estimating the sensitivity function in double logarithmic format. The study revealed that the estimated planted area in the current year, during the period under study is increasing by $3.65 \%$. and $1.61 \%$ if the Erdab and net acre returns of wheat price escalated by the real values in the previous year by $10 \%$. Whereas, it is decreased by $1.92 \%$ if the production costs are increased by the real values of the previous year $10 \%$. If all parameters of the function were to be increased by $10 \%$, the expected planted area in the current year within the period under study would increase by $3.34 \%$, which is statistically confirmed at known economical standards. Therefore, it is important to take into consideration the consequences of increasing agricultural prices and production costs, and net Feddan returns when deciding production strategies related to wheat production in the upcoming years where the production strategies would be decided.

In light of the above, the study recommends the following:

1- Continue increasing agricultural prices in accordance to the economical situations of the country, especially inflation.

2- Publishing crop prices by all means of advertising before planting crops by a reasonable time period. So that agricultures would be motivated to plant wheat aimed areas.

3- Concern not to increase the production costs. This could be achieved by saving all necessities of production in the appropriate time and achievable prices for the majority of agricultures.

4- Interest in the deployment of high productivity species and its agricultural extention methods that have a net positive Feddan return.

Fayoum J. Agric. Res. \& Dev., Vol.25, No.2, July, 2011 
rq

5- Speeding up issuing of the new cooperation law, where it contains providing and activating the basic roles for production and marketing and production cooperation.

Fayoum J. Agric. Res. \& Dev., Vol.25, No.2, July, 2011 